\title{
Competing Long-Range Bonds and Site Dilution in the One-Dimensional Bond-Percolation Problem
}

\author{
U. L. Fulco ${ }^{1}$, L. R. da Silva ${ }^{2}$, F. D. Nobre ${ }^{2}$, and L. S. Lucena ${ }^{2}$ \\ ${ }^{1}$ Departamento de Física, Universidade Federal do Piauí, 64049-550, Teresina-PI, Brazil \\ ${ }^{2}$ Departamento de Física Teórica e Experimental, \\ Universidade Federal do Rio Grande do Norte, 59072-970, Natal-RN, Brazil
}

Received on 30 May, 2003

\begin{abstract}
The long-range bond-percolation problem, on a linear chain $(d=1)$, in the presence of diluted sites (with an occupancy probability $p_{s}$ for an active site) is studied by means of a Monte Carlo simulation. The occupancy probability for a bond between two active sites $i$ and $j$, separated by a distance $r_{i j}$ is given by $p_{i j}=p / r_{i j}^{\alpha}$, where $p$ represents the usual occupancy probability between nearest-neighbor sites. This model allows one to analyse the competition between long-range bonds (which enhance percolation) and diluted sites (which weaken percolation). By varying the parameter $\alpha(\alpha \geq 0)$, one may find a crossover between a nonextensive regime and an extensive regime; in particular, the cases $\alpha=0$ and $\alpha \rightarrow \infty$ represent, respectively, two well-known limits, namely, the mean-field (infinite-range bonds) and first-neighbor-bond limits. The percolation order parameter, $P_{\infty}$, was investigated numerically for different values of $\alpha$ and $p_{s}$. Two characteristic values of $\alpha$ were found, which depend on the site-occupancy probability $p_{s}$, namely, $\alpha_{1}\left(p_{s}\right)$ and $\alpha_{2}\left(p_{s}\right)\left(\alpha_{2}\left(p_{s}\right)>\alpha_{1}\left(p_{s}\right) \geq 0\right)$. The parameter $P_{\infty}$ equals unit, $\forall p>0$, for $0 \leq \alpha \leq \alpha_{1}\left(p_{s}\right)$ and vanishes, $\forall p<1$, for $\alpha>\alpha_{2}\left(p_{s}\right)$. In the interval $\alpha_{1}\left(p_{s}\right)<\alpha<\alpha_{2}\left(p_{s}\right)$, the parameter $P_{\infty}$ displays a familiar behavior, i.e., 0 for $p \leq p_{c}(\alpha)$ and finite otherwise. It is shown that both $\alpha_{1}\left(p_{s}\right)$ and $\alpha_{2}\left(p_{s}\right)$ decrease with the inclusion of diluted sites. For a fixed $p_{s}$, it is shown that a convenient variable, $p^{*} \equiv p^{*}(p, \alpha, N)$, may be defined in such a way that plots of $P_{\infty}$ versus $p^{*}$ collapse for different sizes and values of $\alpha$ in the nonextensive regime.
\end{abstract}

\section{Introduction}

Percolation [1, 2] represents one of the most interesting problems in statistical physics. Many physical situations depend essentially on the geometric properties of random clusters, e.g., random resistor networks, forest fires, and the flow of fluids in porous media. The study of such clusters, and, in particular, the existence of an infinite connected cluster which spans the system in question, is the subject of percolation theory. The percolation order parameter, $P_{\infty}$, is defined as the fraction of sites of the system that belong to the infinite cluster. Obviously, $P_{\infty}$ attains its maximum value $\left(P_{\infty}=1\right)$ when all the sites of the system appear inside the infinite cluster, whereas $P_{\infty}=0$ below a certain threshold, when it is not possible to produce an infinite cluster. For the bond-percolation problem, where $p$ represents the usual occupancy probability between nearest-neighbor sites, the one-dimensional problem is trivial: one has that $P_{\infty}=1$ for $p=1$ and $P_{\infty}=0 \forall p<1$. Also, if one introduces a dilution of sites in the system (with an occupancy probability $p_{s}$ for an active site), one gets that $P_{\infty}=0$ (even for $p=1$ ) $\forall p_{s}<1$. However, this trivial situation changes completely if one introduces long-range effects, i.e., bond-occupancy probabilities associated with two active sites further than nearest-neighboring ones. An interesting competition may occur in such a case between the dilution of sites and the range of the bond-occupancy probability. It is important to mention that site-diluted long-range bond-percolation models are closely related to real situations in a wide variety of complex networks, such as internet, www, small-world economic networks [3, 4], and the network of the Brazilian Popular Music [5]. Due to the presence of long-range bond occupancy probabilities, such a system may also present interesting nonextensive behavior $[4,6]$.

The extensivity of some thermodynamical quantities, like the internal and free energies, is one of the main properties of the standard Boltzmann-Gibbs statistical-mechanics formalism [7, 8, 9]. Within such a formalism, the extensive quantities increase linearly with the size of the system (e.g., the number of particles, $N$ ), in such a way that the internal energy per particle, or the free-energy per particle, approach well-defined values (independent of $N$ ) in the thermodynamic limit. However, only a few textbooks [10] emphasize that the extensivity property does not hold in general, being directly related to the characteristics of the system under consideration, e.g., the interactions among particles must be short-ranged. When long-range interactions are present, each particle interacts with all the others, leading to a free energy that depends more than linearly on $N$, and obviously, the extensivity property looses its validity. As a consequence of this, additivity does not hold, in the sense that the total free energy is not equal to the sum of the free energies of the macroscopic parts of the system.

A lot of interest has been dedicated to the study of 
nonextensive systems lately. Nonextensive behavior has been found in many physical systems, and may be produced by a variety of physical ingredients, like long-range interactions and long-time memory, among others. Clear limitations have been identified on standard formalisms, e.g., the powerful Boltzmann-Gibbs statistical mechanics was shown to fail in providing a satisfactory description of a wide variety of theoretical models and experimental realizations $[11,12,13]$, in such a way that a completely new physics is emerging for dealing with such systems, in the recent years. In order to deal with such systems, the most successful alternative for the standard Boltzmann-Gibbs formalism, so far, appears to be the nonextensive thermostatistics, due to Tsallis, based on the generalized entropic form [14]

$$
S_{q}=k \frac{\left[1-\sum_{i=1}^{W} p_{i}^{q}\right]}{q-1},
$$

where $k$ is a positive constant, $W$ represents the total number of microscopic possibilities and $p_{i}$ stands for the occurrence probability of the microscopic configuration $i$. The entropic index $q$ (for which the particular case $q=$ 1 recovers the standard Boltzmann-Gibbs entropy, $S_{1}=$ $-k \sum_{i=1}^{W} p_{i} \ln p_{i}$ ) is responsible for the nonextensivity in the thermodynamic quantities. For instance, if one considers two independent systems, $A$ and $B$, one gets that,

$$
S_{q}(A+B)=S_{q}(A)+S_{q}(B)+(1-q) S_{q}(A) S_{q}(B),
$$

which breaks the usual additivity property of the entropy for any $q \neq 1$.

Clear evidence of breakdown of ergodicity has been found recently on Hamiltonian systems with long-range interactions $[15,16,17,18,19,20]$, through the appearance of a metastable state, before the terminal equilibrium state is attained. For a finite number of particles, if one waits a sufficiently long time, the system experiences a crossover from the metastable state to a terminal equilibrium state, that presents some properties in agreement with the usual Boltzmann-Gibbs formalism. However, the metastable-state survival time increases with the size of the system, in such a way that if one performs the thermodynamic limit before the long-time limit, the system will remain in this regime forever. Therefore, since the terminal equilibrium may never be attained, one needs an appropriate statistical-mechanics formalism to deal with such a metastable state; in fact, some of its physical properties are in agreement with Tsallis's prescription. However, for a finite number of particles, the system displays a crossover from the metastable state (where the nonextensive thermodynamics seems to apply) to the terminal equilibrium state (where extensivity is recovered) $[18,19]$.

A crossover between nonextensive and extensive regimes has been found also in the long-range onedimensional bond-percolation problem [6]. In this problem, one considers a bond occupancy probability between any two sites $i$ and $j$ of the linear chain as,

$$
p_{i j}=\frac{p}{r_{i j}^{\alpha}} ; \quad(0 \leq p \leq 1 ; \alpha \geq 0),
$$

where $r_{i j}$ represents the distance between sites (in crystalline units, $\left.r_{i j}=0,1,2, \cdots\right)$ and $p$ denotes the firstneighbor bond occupancy probability. The exponent $\alpha$ controls the range of the bonds in the chain, yielding two wellknown particular cases, namely, the first-neighbor $(\alpha \rightarrow \infty)$ and infinite-range, i.e., mean-field-like $(\alpha=0)$, bondpercolation limits. Such a crossover was found to occur at $\alpha \equiv \alpha_{1}=1$, in such a way that for $0 \leq \alpha \leq \alpha_{1}$ (nonextensive regime) the percolation order parameter is always maximum. The extensive regime holds for all $\alpha>\alpha_{1}$, although only for $\alpha>\alpha_{2}\left(\alpha_{2}=2\right)$, the percolation order parameter presents the usual behavior characteristic of the one-dimensional first-neighbor bond-percolation, vanishing $\forall p<1$. In the interval $\alpha_{1}<\alpha<\alpha_{2}$, the order parameter presents a nontrivial behavior, varying with $p$ between its minimum and maximum possible values.

By introducing a site dilution [probabilities $p_{s}$ and $\left(1-p_{s}\right)$ for a site to be present or vacant, respectively], in the above-mentioned long-range one-dimensional bondpercolation problem [4], we shall investigate herein the competition between the long-range bonds and the site dilution. In particular, the values of $\alpha$ defining the nonextensive and extensive regimes, will now depend on $p_{s}$, i.e., $\alpha_{1} \equiv \alpha_{1}\left(p_{s}\right)$ and $\alpha_{2} \equiv \alpha_{2}\left(p_{s}\right)$. In the next section we present and discuss our results; finally, in section 3 we present our conclusions.

\section{Results and Discussion}

We have simulated open linear chains of several sizes varying from $N=100$ up to $N=1000$; in each case, we have computed $P_{\infty}$ by considering averages over 10000 samples (i.e., different configurations of bonds and diluted sites).

The percolation order parameter, $P_{\infty}(p)$, for typical values of $\alpha, p_{s}$ and $N$ is presented in Figs. 1-3. In Fig. 1 we show $P_{\infty}(p)$ for fixed values of $\alpha(\alpha=0.8)$ and $N(N=$ $300)$, and several values of $p_{s}\left(p_{s}=0.8,0.6,0.4,0.2\right)$. One sees clearly that the order parameter is weakened by the dilution of sites, leading to an increase in the critical value of the first-neighbor bond occupancy probability, $p_{c}$, below which $P_{\infty}$ becomes zero. As expected, fluctuations get enlarged, for higher fractions of diluted sites (cf. the case $p_{s}=0.2$ in Fig. 1), in such a way that a larger computational effort is required in order to get reliable results in such cases. In Fig. 2 the order parameter is exhibited for fixed values of $p_{s}$ $\left(p_{s}=0.8\right)$ and $N(N=300)$ and several values of $\alpha$, in the range $\alpha=0.2$ up to $\alpha=2.0$. One notices that, for small values of $\alpha$ one is found in the nonextensive regime, for which $P_{\infty}$ equals unit, $\forall p>0$, leading to $p_{c}(\alpha)=0$. However, for increasing values of $\alpha$, one gets $p_{c}(\alpha)>0$, in such a way that as one approaches the first-neighbor bond-percolation limit (typically, $\alpha \sim 2$ ), large fluctuations are observed, due to finite size effects; in such a case, one should get, in the thermodynamic limit, $P_{\infty}=0$ and $p_{c}(\alpha)=1$. In Fig. 3 we show how the order parameter evolves with the size of the system; we consider two different site concentrations $\left(p_{s}=0.6\right.$ and $\left.p_{s}=0.2\right)$ and a fixed value of $\alpha(\alpha=0.8)$. Once again, one observes that the finite-size effects become 
more relevant for lower site concentrations (cf. the different sizes considered for the case $p_{s}=0.2$ of Fig. 3), in such a way that more reliable estimates of $P_{\infty}$ and $p_{c}(\alpha)$ are obtained at large system sizes (typically, $N=1000$ ). By locating, for finite values of $N$, the abcissa points given by the linear continuations of the curves of $P_{\infty}(p)$, and then extrapolating for $N \rightarrow \infty$, one may estimate the critical values of the first-neighbor bond occupancy probability, for fixed values of $p_{s}$ and different values of $\alpha, p_{c}(\alpha)$.

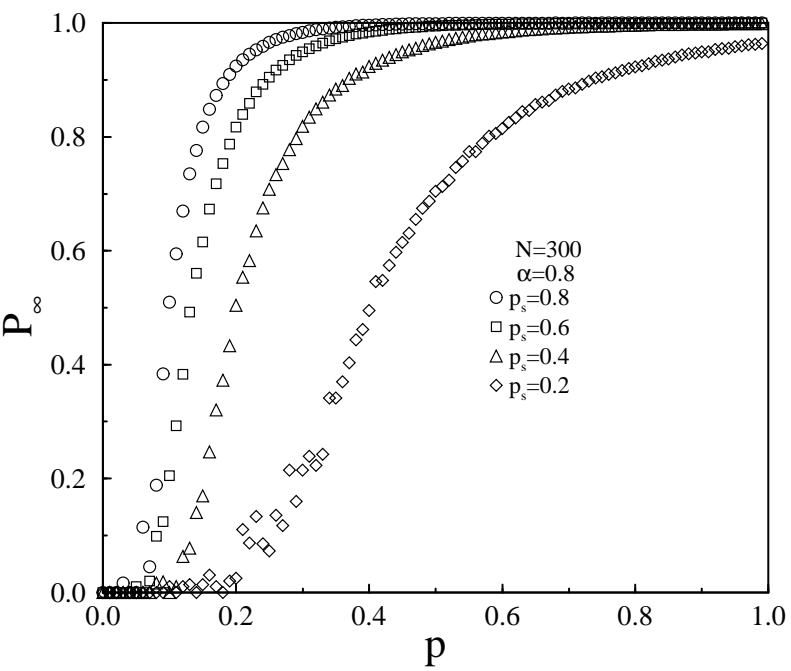

Figure 1. The percolation order paramenter, $P_{\infty}$, versus the firstneighbor bond-percolation occupancy probability, $p$, for fixed values of $N$ and $\alpha$, and several values of the site-occupancy probability $p_{s}$.

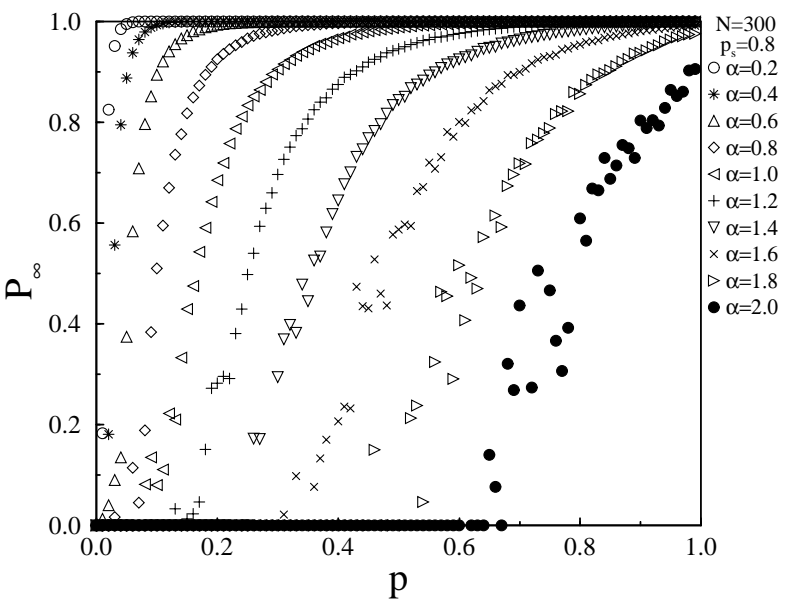

Figure 2. The percolation order paramenter, $P_{\infty}$, versus the firstneighbor bond-percolation occupancy probability, $p$, for fixed values of $N$ and $p_{s}$, and typical values of $\alpha$.

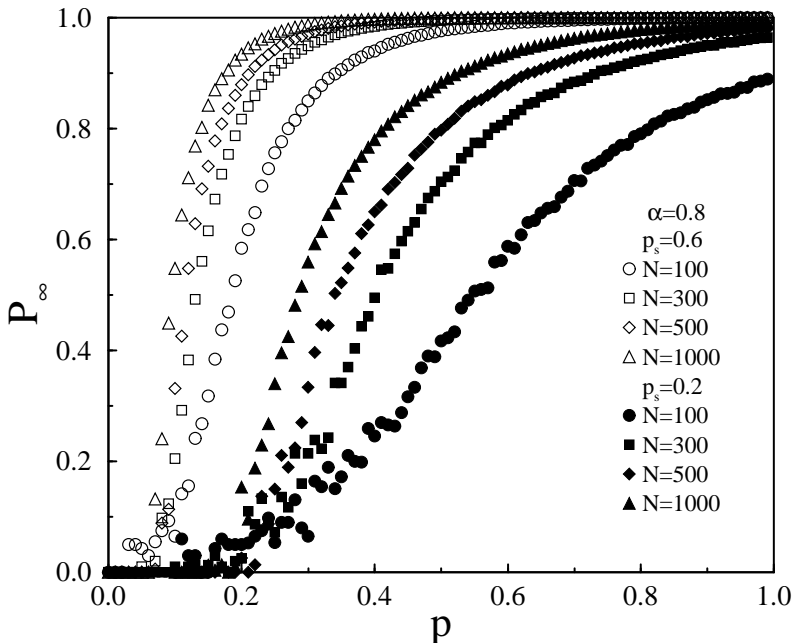

Figure 3. The percolation order paramenter, $P_{\infty}$, versus the firstneighbor bond-percolation occupancy probability, $p$, for a fixed value of $\alpha$, two different site-occupancy probabilities, and several system sizes.

In Fig. 4 we exhibit how $p_{c}(\alpha)$ varies with $\alpha$ for a typical value of the site concentration: $p_{s}=0.6$. The error bars express the accuracy of the linear continuations, together with the thermodynamic-limit extrapolations, of the curves of the parameter $P_{\infty}$. By comparing the results of Fig. 4 with those obtained for different site concentrations, one notices that the two characteristic values of $\alpha, \alpha_{1}\left(p_{s}\right)$, below which $P_{\infty}$ equals unit, $\forall p>0$, and $\alpha_{2}\left(p_{s}\right)$, above which $P_{\infty}=0, \forall p<1$, decrease as the site concentration decreases. In analogy to what happens in the case of no site dilution $\left(p_{s}=1.0\right)$ [6], the characteristic value $\alpha_{1}\left(p_{s}\right)$ sets in a crossover between the nonextensive $\left[0 \leq \alpha \leq \alpha_{1}\left(p_{s}\right)\right]$ and extensive $\left[\alpha>\alpha_{1}\left(p_{s}\right)\right]$ regimes. On the other hand, $\alpha_{2}\left(p_{s}\right)$ defines the onset of the well-known first-neighbor one-dimensional percolation behavior; as a consequence of this, the region right below $\alpha_{2}\left(p_{s}\right)$ is very delicate from the numerical point of view. In Fig. 4, the last point to the right corresponds to the estimate of $p_{c}(\alpha)$ associated with the value of $\alpha$ that is as close as possible (in the sense that one may still get a reliable estimate, within the computational effort employed herein) to $\alpha_{2}\left(p_{s}\right)$. For slightly higher values of $\alpha$, strong fluctuations, driven by finite-size effects, occur; in this region, in order to observe the behavior of the order parameter $P_{\infty}$ (i.e., whether it drops to zero $\forall p<1$, or not) one needs to run much larger system sizes, as well as to increase reasonably the number of samples, as compared to those used in the present work. Besides the site-occupancy probability $p_{s}=0.6$, we have also investigated the cases $p_{s}=0.4$ and $p_{s}=0.8$; the corresponding plots are similar to the one exhibited in Fig. 4, except that the characteristic values $\alpha_{1}\left(p_{s}\right)$ and $\alpha_{2}\left(p_{s}\right)$ are different. Below, we present our estimates for the two characteristic values of $\alpha$, for each one of site concentrations considered,

$p_{s}=0.8: \quad \alpha_{1}\left(p_{s}\right)=0.7 \pm 0.05 ; \quad \alpha_{2}\left(p_{s}\right)=1.9 \pm 0.06 ;$

$p_{s}=0.6: \quad \alpha_{1}\left(p_{s}\right)=0.6 \pm 0.07 ; \quad \alpha_{2}\left(p_{s}\right)=1.8 \pm 0.07 ;$

$p_{s}=0.4: \quad \alpha_{1}\left(p_{s}\right)=0.5 \pm 0.09 ; \quad \alpha_{2}\left(p_{s}\right)=1.7 \pm 0.10$.

The above results, together with those for the case $p_{s}=1.0$, i.e., $\alpha_{1}\left(p_{s}\right)=1.0$ and $\alpha_{2}\left(p_{s}\right)=2.0$ [6], do surely indicate 
that the characteristic values of $\alpha$ decrease with the concentration of sites.

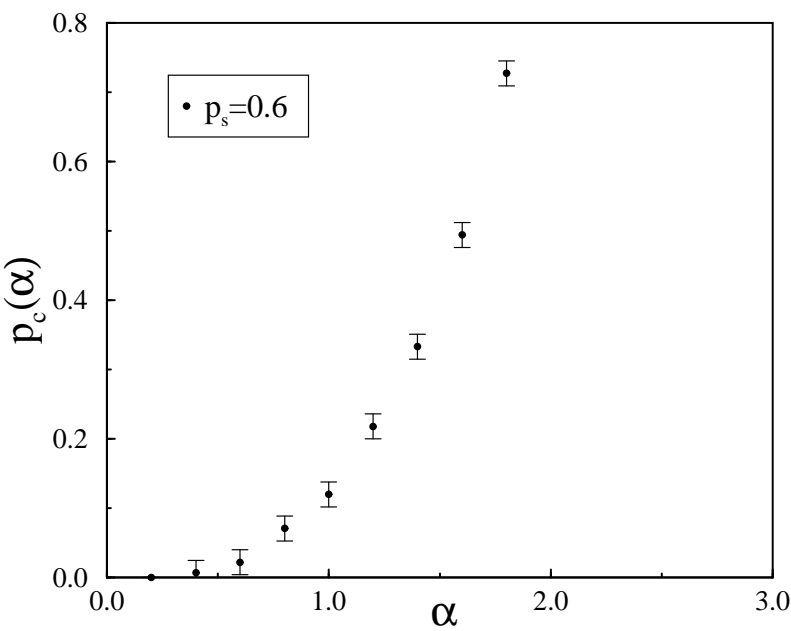

Figure 4. The critical value of the first-neighbor bond occupancy probability versus $\alpha$, for the case $p_{s}=0.6$.

We shall now concentrate on small values of $\alpha$ (roughly speaking, values of $\alpha$ inside the nonextensive region); let us define a rescaled occupancy probability, $p^{*}$,

$$
1-p^{*}=(1-p)^{N^{*} 2^{\alpha}} ; \quad N^{*} \equiv \frac{N^{1-\alpha}-1}{1-\alpha} .
$$

By considering the percolation order parameter in terms of such a variable, i.e., $P_{\infty}\left(p^{*}\right)$, we have verified that, for a fixed value of $p_{s}$, the curves associated with different values of $\alpha$ and $N$ collapse. In Fig. 5, where we exhibit curves for the typical site concentration $p_{s}=0.6$, one sees a reasonably good data collapse (except for the vicinity of the critical point). Therefore, one may define a critical rescaled occupancy probability which depends on the site occupancy, $p_{c}^{*} \equiv p_{c}^{*}\left(p_{s}\right)$, below which $P_{\infty}\left(p^{*}\right)=0$. It should be mentioned that our data collapse works for values of $\alpha$ below, or slightly above, the characteristic value $\alpha_{1}\left(p_{s}\right)$, but not for values of $\alpha$ far above $\alpha_{1}\left(p_{s}\right)$, i.e., far inside the extensive region. We have also worked out data collapses for the site concentrations $p_{s}=0.4$ and $p_{s}=0.8$, leading to plots similar to those of Fig. 5. The estimated critical rescaled occupancy probabilities are listed below,

$$
\begin{array}{ll}
p_{s}=0.8: & p_{c}^{*}\left(p_{s}\right)=0.6 \pm 0.05 ; \\
p_{s}=0.6: & p_{c}^{*}\left(p_{s}\right)=0.7 \pm 0.04 ; \\
p_{s}=0.4: & p_{c}^{*}\left(p_{s}\right)=0.8 \pm 0.05,
\end{array}
$$

which, at least for the values of $p_{s}$ investigated, decrease with $p_{s}$ according to a straight line. Therefore, in analogy to what has been verified for the case $p_{s}=1$ [6], the nonextensive region may be unified in terms of a conveniently defined variable.

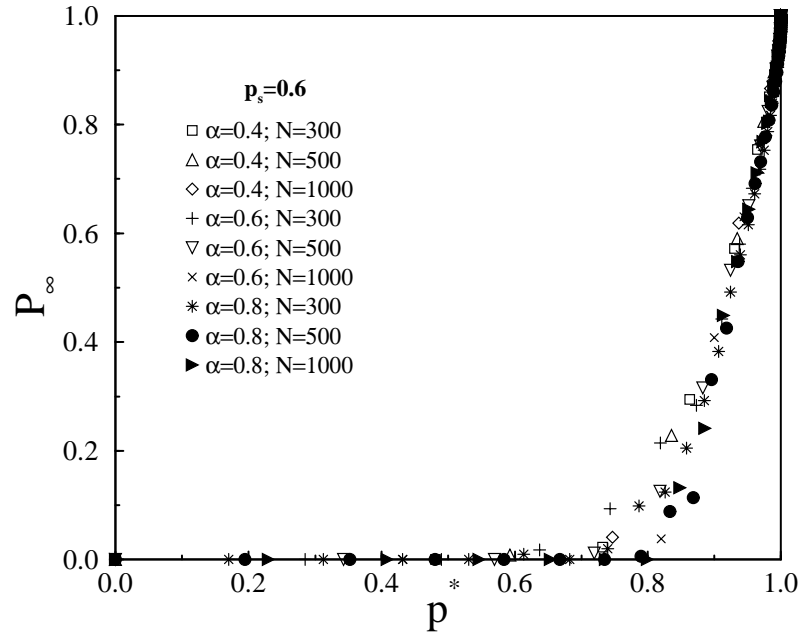

Figure 5. Data collapse of curves for different values of $N$ and $\alpha$, of the order parameter $P_{\infty}$ as a function of the variable $p^{*}$, for the case $p_{s}=0.6$.

\section{Conclusion}

We have investigated the competition between the dilution of sites (with a site occupancy probability $p_{s}$ ) and longrange bonds in the one-dimensional bond-percolation problem, by means of Monte Carlo simulations. The range of the bonds is controlled by a parameter $\alpha$, in such a way that two well-known limits may be recovered, namely, the mean-field $(\alpha=0)$, and the first-neighbor bond percolation $(\alpha \rightarrow \infty)$ ones. We have shown that two characteristic values of $\alpha$ occur, whose values depend on the site-occupancy probability, $\alpha_{1}\left(p_{s}\right)$ and $\alpha_{2}\left(p_{s}\right)\left[\alpha_{2}\left(p_{s}\right)>\alpha_{1}\left(p_{s}\right) \geq 0\right.$ ]. For $0 \leq \alpha \leq \alpha_{1}\left(p_{s}\right)$, the percolation order parameter $P_{\infty}$ is always maximum (equal to unit), indicating that one is in a nonextensive regime, whereas for $\alpha>\alpha_{2}\left(p_{s}\right)$, the order parameter is always zero, in such a way that one is found in the well-known first-neighbor one-dimensional bond-percolation regime. The crossover between the nonextensive and extensive regimes takes place at $\alpha=\alpha_{1}\left(p_{s}\right)$ and for $\alpha_{1}\left(p_{s}\right)<\alpha<\alpha_{2}\left(p_{s}\right)$ the order parameter displays a familiar behavior, i.e., varying monotonically between its minimum and maximum possible values. We have shown that the two characteristic values of $\alpha, \alpha_{1}\left(p_{s}\right)$ and $\alpha_{2}\left(p_{s}\right)$, decrease with the inclusion of diluted sites. Finally, we have verified that, for the nonextensive region, it is possible to define a convenient variable in such a way that curves for fixed values of $p_{s}$ are unified.

\section{Acknowledgments}

It is a pleasure to thank D. Stauffer, C. Tsallis, and H. H. A. Rego for fruitful conversations. The partial financial supports from CNPq, CAPES, Pronex/MCT, and ANP/CTPETRO/CENPES/Petrobras (Brazilian agencies) are acknowledged. 


\section{References}

[1] D. Stauffer and A. Aharony, Introduction to Percolation Theory, Second Edition (Taylor and Francis, London, 1994).

[2] G. Grimmet, Percolation, Second Edition (Springer Verlag, Berlin, 1999).

[3] G. Corso, L. S. Lucena, and Z. D. Thomé, Physica A 324, 430 (2003).

[4] U. L. Fulco, L. R. da Silva, F. D. Nobre, H. H. A. Rego, and L. S. Lucena, Phys. Lett. A, 312, 331 (2003).

[5] D. L. Silva, M. M. Soares, M. V. C. Henriques, M. T. Schivani, S. G. Aguiar, T. P. Carvalho, G. Corso, and L. S. Lucena, to appear in Physica A.

[6] H. H. A. Rego, L. S. Lucena, L. R. da Silva, and C. Tsallis, Physica A 266, 42 (1999).

[7] R. K. Pathria, Statistical Mechanics, (Butterworth, Washington DC, 1996).

[8] H. E. Stanley, Introduction to Phase Transitions and Critical Phenomena, (Oxford University Press, New York, 1971).

[9] P. T. Landsberg, Thermodynamics and Statistical Mechanics, (Dover, New York, 1991).

[10] R. Balescu, Equilibrium and Nonequilibrium Statistical Mechanics, (Wiley, New York, 1974).
[11] C. Tsallis, Brazilian Journal of Physics 29, 1 (1999).

[12] C. Tsallis, in Nonextensive Statistical Mechanics and its Applications, edited by S. Abe and Y. Okamoto, Lecture Notes in Physics (Springer-Verlag, Berlin, 2001).

[13] C. Tsallis, in Classical and Quantum Complexity and Nonextensive Thermodynamics, edited by P. Grigolini, C. Tsallis, and B. J. West, Chaos, Solitons and Fractals, 13, 371 (2002).

[14] C. Tsallis, J. Stat. Phys. 52, 479 (1988).

[15] C. Anteneodo and C. Tsallis, Phys. Rev. Lett. 80, 5313 (1998).

[16] A. Campa, A. Giansanti, D. Moroni, and C. Tsallis, Phys. Lett. A 286, 251 (2001).

[17] A. Campa, A. Giansanti, and D. Moroni, Physica 305, 137 (2002).

[18] V. Latora, A. Rapisarda, and C. Tsallis, Phys. Rev. E 64, 056134 (2001)

[19] V. Latora, A. Rapisarda, and C. Tsallis, Physica A 305, 129 (2002).

[20] F. D. Nobre and C. Tsallis, to appear in Phys. Rev. E. 